\title{
Design and Simulation of a Hybrid Silicon/ Electro-optic Polymer Modulator
}

\author{
Kjersti Kleven and Scott T. Dunham \\ University of Washington, Department of Electrical Engineering \\ Seattle, Washington 98195-2500
}

\begin{abstract}
The design and simulation of a novel resonant cavity optical modulator is presented in this work. The device incorporates a hybrid silicon/electro-optic polymer waveguide in the cavity region with a distributed Bragg reflector in a single mode silicon waveguide on each side of the cavity to create a modulator with a large modulation depth, short device length and a low drive voltage for use in future high speed integrated optics applications. Full 3D simulations of this device structure are very computationally expensive; therefore the periodicity of the device has been exploited to allow for a cascade matrix approach to be employed to reduce the time/computational resources necessary for accurate simulation of light propagation during the optimization phase of the device design.
\end{abstract}

\section{INTRODUCTION}

Many research groups have demonstrated the use of silicon in Mach-Zehnder modulator devices. However, the length of silicon modulators is generally quite long, and it is challenging to achieve high-speed optical devices in silicon [1]. Recent advances in electro-optic (EO) polymer design have resulted in fast response polymers with large EO coefficients $(\sim 100 \mathrm{pm} / \mathrm{V})$, which allow for high speed switching with lower applied voltages [2]. The structure proposed here combines the benefits of the high index of refraction and manufacturability of silicon with the strong, high speed response of the EO polymer in a hybrid waveguide resonant cavity modulator to create a high-speed device with a low drive voltage, large modulation depth, and compact size. The analysis uses an alternative modeling approach to significantly reduce the computational resources necessary to perform $3 \mathrm{D}$ analysis, which is critical for device design and optimization.

\section{HYBRID WAVEGUIDE STRUCTURE}

This work looks specifically at a resonant cavity modulator design incorporating a hybrid silicon/EO polymer slot waveguide in the cavity region with distributed reflectors in the silicon waveguide, as shown in Fig 1. Almeida et al. have described a waveguide in which the light can be guided through a void nanostructure if there are narrow high index ridges on each side of the void region [3]. The intensity of the light is enhanced in the void region due to the boundary conditions enforced by Maxwell's equations at the interfaces. If a low index EO polymer is used instead of a void region, the field will be similar to that of the void/silicon waveguide. However, this alternative structure also allows a field to be applied across the EO polymer region to cause a change in the propagation constant of the silicon/polymer waveguide, thereby modulating the output intensity at a given wavelength in a resonant cavity modulator.

The confinement of light in the low index region is generally not what would be expected, as light is normally confined to the higher index material. However, when the dimensions of the high index material are such that they do not allow for individual guided modes, the solution to Maxwell's equations results in a hybrid mode. The differences in indices of refraction between the silicon and EO polymer result in a field that is highly confined to the polymer region in the hybrid waveguide, as illustrated in Figs. 2 and 3. An effective index approach has been used to estimate the achievable change in propagation constant, with $\beta_{\text {eff }}=k_{o}{ }^{*} s q r t\left[N_{p}{ }^{2}+b_{e q} *\left(N_{\text {hybrid }}{ }^{2}-N_{p}{ }^{2}\right)\right]$. This estimate was compared to a finite element simulation, and the change of propagation constant varied up to $20 \%$ from the finite element solution, thereby verifying the need for $3 \mathrm{D}$ simulation. The device dimensions were optimized to achieve the largest change in the propagation constant for a given change in the index of refraction of the EO polymer, and these dimensions were then used as the cavity region in the subsequent modulator design.

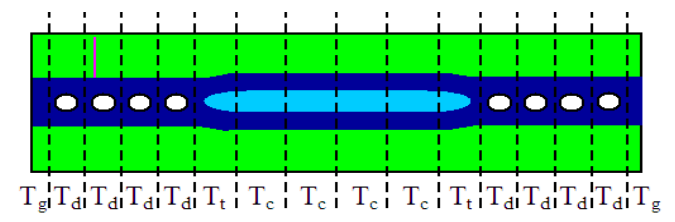

Fig. 1. Top view of resonant cavity modulator. Dark blue is silicon and light blue is the EO polymer in the hybrid waveguide structure. Sections used for the cascade matrix simulation are shown.

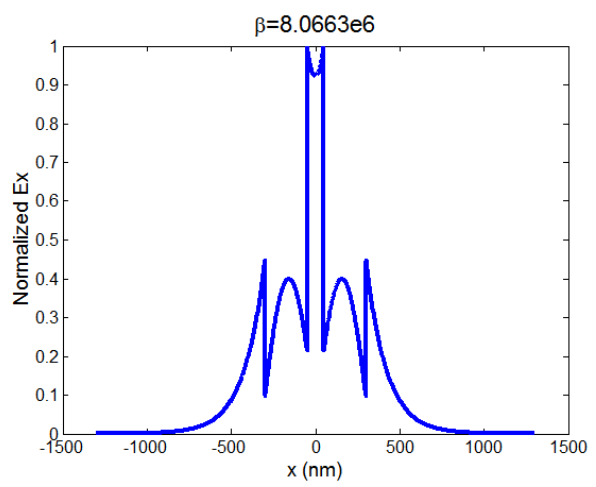

Fig. 2. Field profile for a $100 \mathrm{~nm}$ polymer core with $250 \mathrm{~nm}$ silicon ridges as calculated with the effective index approach. 


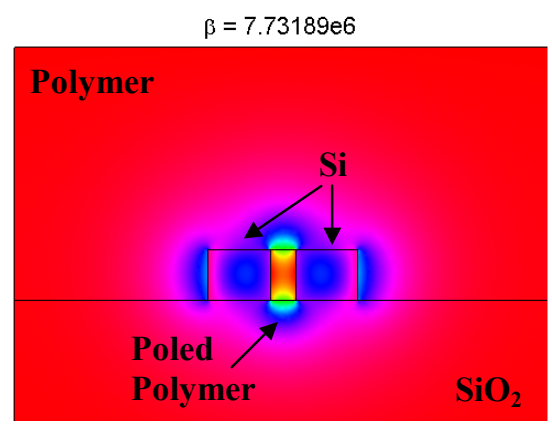

Fig. 3. Cross section of field profile from a finite element simulation for a $100 \mathrm{~nm}$ polymer core with $250 \mathrm{~nm}$ silicon ridges showing enhanced field intensity in the core.

\section{DEVICE ANALYSIS \& SIMULATION}

Due to the complex nature of the structure, high operating frequencies, and small device dimensions, 3D simulation is required to obtain accurate propagation characteristics, particularly scattering losses. However, full wave simulation of this device is very computationally expensive, especially for design optimization. This work takes advantage of the periodicity of the resonant cavity structure by utilizing cascade matrices to reduce simulation times [4]. With this approach, only the small unique sections of the device need to be simulated separately with $3 \mathrm{D}$ full wave modeling. The entire device structure can then be modeled using matrix multiplication of the transmission characteristics $(\boldsymbol{T})$ of the individual sections in the appropriate order, as shown in Eq. (1) and Fig. 1. A comparison of the simulations of a four-hole periodic bandgap structure is shown in Fig. 4, which demonstrates excellent agreement between the full structure simulations and the cascade matrix approach.

$$
\vec{T}=\vec{T}_{g} \vec{T}_{d} \vec{T}_{d} \vec{T}_{d} \vec{T}_{d} \vec{T}_{t} \vec{T}_{c} \vec{T}_{t} \vec{T}_{d} \vec{T}_{d} \vec{T}_{d} \vec{T}_{d} \vec{T}_{g}
$$

The reduction in computation time for this device configuration is very significant because the entire device can be analyzed and optimized with only a small set of simulations, and the effect of parameters such as the number of defects, cavity length, and defect spacing can be analyzed without performing additional simulations. It is also possible to easily

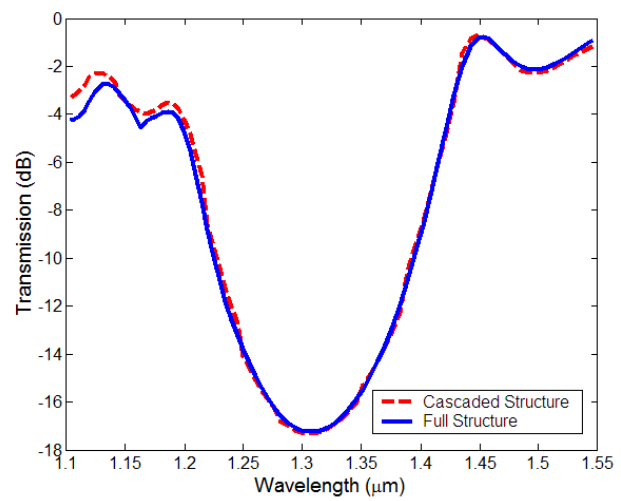

Fig. 4. Comparison of transmission through a photonic bandgap structure for both the cascaded matrix approach and the full structure simulation. The agreement could be improved further below $1.15 \mu \mathrm{m}$ by including the second propagating mode in a higher order matrix representation.

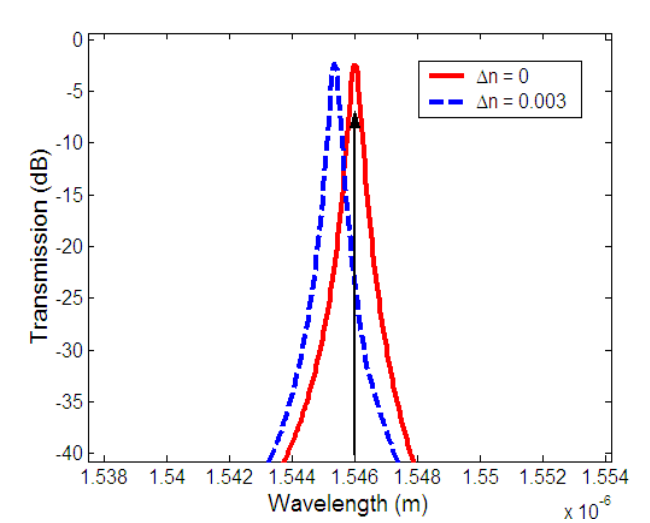

Fig. 5. Simulated transmission of a 5-period DBR with a $20 \mu \mathrm{m}$ cavity region showing a $91 \%$ modulation depth.

incorporate design changes using this method; a change in defect shape, type of cavity structure, or defect size requires only that section to be simulated again rather than whole device.

Simulated transmission through a device with 5 period reflectors on each side and a $20 \mu \mathrm{m}$ cavity is shown in Fig. 5. The total device length for this modulator is only $28 \mu \mathrm{m}$, compared to a Mach-Zehnder structure with the hybrid waveguide, which would need an active length of $550 \mu \mathrm{m}$ for full modulation. The FWHM of this configuration is $0.4 \mathrm{~nm}$ and a modulation depth of $91 \%$ can be achieved with a refractive index change of only -0.003 , which corresponds to an applied voltage of $3 \mathrm{~V}$ with the silicon ridges in the cavity region serving as electrodes. Design trade-offs can be made by varying the number of reflector periods and the length of the resonant cavity to improve either the maximum transmission or FWHM, depending upon the application.

This resonant cavity modulator design results in a large modulation depth and small FWHM for a very short overall device length. By incorporating the hybrid waveguide structure in the resonant cavity region, only a small voltage is necessary to cause a significant change in the propagation constant and therefore shift the resonant wavelength of the device. Recognizing the periodicity of the device structure and using cascade matrices reduces the required simulation times dramatically and provides an approach that could be used for optimization of many integrated optics devices.

\section{REFERENCES}

[1] Liu, R. Jones, L. Liao, D. Samara-Rubio, D. Rubin, O. Cohen, R. Nicolaescu, and M. Paniccla, "A high-speed silicon optical modulator based on a metal-oxide-semiconductor capacitor," Nature, vol. 427, pp. 615-618, Feb. 2004.

[2] N. Tucker, H. Li, H. Tang, L. R. Dalton, Y. Liao, B. H. Robinson, A. K. Jen, J. Luo, S. Liu, M. Haller, J. Kang, T. Kim, S. Jang, B. Chen, "Recent Progress in Developing Highly Efficient and Thermally Stable Nonlinear Optical Polymers for Electro-Optics," Proc. SPIE, Vol 5351, $36-43,2004$.

[3] V.R. Almeida, Q. Xu, C.A. Barrios, and M. Lipson, "Guiding and confining light in void nanostructures," Optics Lett. vol. 29, pp. 12091211, June 2004.

[4] D.M. Kearns and R.W. Beatty, Basic Theory of Waveguide Junctions and Introductory Microwave Network Analysis, Pergamon Press, Oxford, London, 1967. 